\title{
Feasibility of Expanding Traffic Monitoring Systems with Floating Car Data Technology
}

\author{
Authors Name: Wim Vandenberghe, Erik Vanhauwaert, Sofie Verbrugge, Ingrid Moerman, Piet Demeester \\ Affiliation: Department of Information Technology (INTEC), Ghent University - IBBT \\ E-mail: wim.vandenberghe@intec.ugent.be
}

Trajectory information reported by certain vehicles (Floating Car Data or FCD) can be applied to monitor traffic states on the road network. However, policy makers face difficulties when deciding to invest in the expansion of their infrastructure based on inductive loops and cameras, or to invest in the roll-out of a FCD system. This paper aims to support this decision. The provided functionality of a FCD system is investigated and the minimum requirements are determined. It is researched if the proposed deployment would introduce any reliability issues. The communication cost is derived and combined with other costs elements to assess the total costs for different implementation scenarios. The outcome of this research is to target a FCD deployment with a penetration rate of 1\%, a sample interval of 10 seconds and a transmission interval of 30 seconds. Such a system will accurately determine the locations of both incidents and the tail end of traffic jams. It can also estimate travel times. This will be very accurate for highways, for arterial and urban roads this will be limited to a binary categorization into normal or congested traffic. No reliability issues are expected. The most cost efficient scenario when deploying a new FCD system is to launch a FCD smartphone application. For Belgium (6 million vehicles), the corresponding cost is 13 million EUR for 10 years. However, it is estimated that purchasing data from companies already acquiring FCD data through their own product could reduce costs with a factor 10 .

Keywords: Floating Car Data, Traffic Monitoring, Intelligent Transport Systems, Feasibility Study. 


\section{Introduction}

The goal of this paper is to determine if the usage of FCD to support traffic monitoring is feasible. This analysis follows a top down approach, defining the research questions from the policy makers' perspective. The Belgian use case receives the primary focus, but the presented results are generic and applicable to any region.

Starting point of this paper is the literature study described in section 2. It provides indications regarding the FCD dataset requirements and supported functionalities. However, these indications are not accurate enough to pronounce on the feasibility of FCD in the context of traffic monitoring. For instance, in a highway environment the minimal penetration rate varies in literature between 1 to $5 \%$. In an urban setting these values are 2 to $10 \%$. Since 6 million vehicles are registered in Belgium, this makes the difference between equipping 60.000 or up to 600.000 vehicles. The former seems feasible, but the latter is quite challenging. This requirement needs to be refined further. The same remark can be made for the sampling interval which greatly influences the amount of communicated data and corresponding cost. To refine these FCD requirements, a simulation platform is used in our research. It is described in section 3. Different scenarios are considered, both in terms of road type and traffic intensities. The platform is used to determine the functionalities that can be supported by FCD data (section 4). The required minimum penetration rate and maximum sample interval are determined in section 5. To assess possible reliability issues, section 6 applies a model that determines the impact of the FCD system on the mobile data network. To investigate the roll out strategies, a cost model is constructed in section 7 .

\section{Related work}

Several technological approaches towards FCD exist. The most commonly applied FCD devices are GPS probes. They are equipped with a GPS receiver and a wireless communication uplink such as GPRS or UMTS. They send their current position and speed to a processing server on a regular basis [1]-[4]. The main advantage of this technique is the accuracy of the measurements and the wide geographical coverage. The disadvantage is the cost, since a GPS probe has to be installed in a relatively large amount of vehicles and a communication cost is introduced. Hence the practical usability of GPS probes strongly depends on the minimal required penetration rate. This defines the amount of vehicles that should be equipped with FCD technology in order to obtain

sufficient accurate application performance. Publications presenting results from Field Operational Tests (FOT) 
did not vary the penetration rate, but applied a fixed rate instead. In the highway environment good results were obtained by Dion \& Rakha [5] with a penetration rate below 1\%, by Bar-Gera [6] with an average penetration rate of $1-3 \%$, by de Fabritiis [2] with a rate of $2.4 \%$, and by Herrera et al. [7] with an average penetration rate of 2-3\%. These results indicate the order of magnitude, but they do not define the exact minimum requirement. Other studies did try to define the exact minimal rate, but the results are diverse. Breitenberger et al. [8] were the only authors to perform an analytical study of the minimal rate, proposing $2.4 \%$ in a highway environment, and $10 \%$ on arterial and urban roads. The other studies relied on simulations. For the highway environment, Chen \& Chien [9] proposed 1\%, Nanthawichit et al. [10] suggested 4 to 5\% and van Lint \& Hoogendoorn [11] obtained 2 to $4 \%$. In an urban setting, Hong et al. [12] proposed 2\% while Mu et al. [13] obtained 10\%. Cheu et al. [14] simulated a mixed scenario, resulting in a minimal rate between 3 and $6 \%$.

Other important topics are the maximum sample interval and the maximum transmission interval of aggregated samples. The former defines the time between two consecutive FCD samples taken by the same floating car. The latter defines the time between two consecutive server uploads by a floating car of all its new samples. When these intervals are too small, unnecessary load on the network and FCD server is introduced, when they are too big, the FCD could become useless. Quiroga and Bullock [1] relied on sampling theory to conclude that the minimum sample interval is at most half the segment travel time. For highways they propose a segment length of 0.2 miles in combination with a sample interval of 6 seconds. However, this analysis is based on the requirement that a single probe should provide a measurement for every segment that it traverses. It does not take into account that the combination of samples from multiple probes could provide the required full coverage. Nanthawichit et al. [10] stated that on highways there is practically no difference between a sample interval of 1 or 10 seconds, that an interval of 30 seconds introduces an acceptable error and that an interval of 60 or 180 seconds is unusable. This study also demonstrated the interaction between penetration rate and sampling interval: in case of a larger interval a higher rate is required to achieve the same accuracy. It concludes that on the highway a penetration rate of 4 to $5 \%$ in combination with a sample interval of 10 to 30 seconds is the minimal configuration. The study of Fontaine \& Smith [15] concluded that when using accurate positioning technology such as GPS, a sample interval of 15 seconds leads to better results than an interval of 60 seconds.

Regarding the maximum transmission interval, Jiang et al. [16] investigated only large transmission intervals: 5, 10 and 15 minutes. They concluded that for a constant sample interval, little difference could be noticed 
between the different transmission intervals. However, this study was limited to traffic without incidents, and only studied travel time estimations. It can be expected that the results are different in more dynamic traffic with changing traffic jam conditions, where delay can greatly influence the performance of applications such as incident detection and traffic jam collision avoidance. Dion et al. [17] proposed techniques that indirectly resulted in a transmission interval between 0 and 92 seconds, with an average of 30 seconds. Satisfactory results were obtained with these intervals, but they were not specifically optimized.

\section{Research environment}

\subsection{Platform}

To refine the FCD system requirements, a simulation platform was developed. As depicted in Figure 1, it consists of 3 different software tools. The first is a microscopic road traffic simulator called SUMO ${ }^{1}$, which is developed by the German Aerospace Center (DLR). During every step of the simulation, the results are written to a trace file. This file is then used as an input for the traffic estimator that was developed by the authors of this paper. It replays the SUMO simulation, treating the data from the trace file as live incoming FCD, and continuously calculates an estimation of the traffic situation. Several parameters can be set: penetration rate, sample interval and the number of previous samples to average. The results are stored in a file that describes the estimated speed for all road segments, for a given amount of points in simulation time. This file is then given to $M_{a t l a b}^{2}$, which depicts the evolution over time of the estimated speed for selected road segments (e.g. Figure 3). It is also possible to depict a snapshot of the estimated traffic situation for all road segments (e.g. Figure 4). On both graphs, conflict zones such as highway entries or traffic lights are indicated by coloured vertical lines.

\subsection{Scenarios}

This paper focuses on the Belgian case study. The simulated scenarios are depicted in Figure 2. Scenario 1 focuses on the highway setting. It represents a trajectory of $44 \mathrm{~km}$ of the E40 highway from Zwijnaarde to Groot-Bijgaarden. It is divided in segments of $500 \mathrm{~m}$. Traffic is categorized in 4 vehicle classes: trucks, slow cars, normal cars and fast cars. Five different traffic intensities were simulated: night-time (a), normal traffic (b),

\footnotetext{
${ }^{1}$ sumo.sourceforge.net
} 
medium congestion (c), heavy congestion (d) and incident (e: sudden blockage of the 2 right lanes between Aalst and Ternat). Scenario 2 represents the urban environment. It represents a trajectory of $7.25 \mathrm{~km}$ of the N9 road, divided in segments of $250 \mathrm{~m}$. It is a typical Belgian road dominated by ribbon development, crossings are manifold, it consists of both single-lane and double-lane segments and is characterized by different speed limits. Traffic was divided equally in slow cars $(\max .50 \mathrm{~km} / \mathrm{h}$ ) and fast cars $(\max .70 \mathrm{~km} / \mathrm{h}$ if allowed). The same intensity scenarios as in scenario 1 are taken into account (a,b,c,d,e:incident blocks the entire road between Exit R4 B and the Kerkstraat).

\subsection{Algorithm}

The goal of this paper is to provide the required insights to support policy makers in their decision of how to expand geographical coverage of their traffic monitoring systems. When investigating this problem, it is of the utter importance that the results are consistent with the currently available FCD technology. It does not make any sense to draw conclusions regarding service level and cost related to beyond state-of-the-art FCD techniques. It is conceivable that positive outcomes of such an analysis would not be met when policy makers would decide to actually adopt commercially available FCD technology. Hence the algorithm used by the traffic estimator was deliberately kept simple compared to recently published FCD algorithms (e.g. van Lint \& Hoogendoorn [11], Cohn [18]). The algorithm is defined as follows:

1. On a regular basis FCD vehicles collect a sample that contains the following information: current coordinates, speed and time. The appropriate values are retrieved from a GPS receiver. This data is sent frequently to a FCD server

2. The FCD server stores a list of received speed values for every road segment. As defined in (1), the length of this list is constant, and determined by the parameter \#samples. It is constructed in a chronological order according the first in - first out principle. During initialization, the list is filled with samples corresponding with the speed limit.

stored_speed_values_segment $\_X=$ speed $_{-} X_{1}$, speed $\_X_{2}, \ldots$, speed $\_X_{\# \text { samples }}$

\footnotetext{
${ }^{2}$ http://www.mathworks.com/products/matlab/
} 
3. The estimation of the current actual speed on a given road segment is then equal to the average of all speeds stored in the corresponding list:

$$
\text { speed_segment_X }=\frac{\sum_{1}^{\# \text { samples }} \text { speed }_{-} X_{i}}{\# \text { samples }}
$$

The parameter \#samples is important: when set too small, the estimated speed will be influenced too much by the samples of a few vehicles, resulting in unwanted fluctuations. If it is too big, detection of congestion will be delayed. To determine the optimum, experiments were conducted both in the highway and the arterial setting for different traffic intensities and different sampling intervals. Based on these experiments, it could be concluded that in general 5 is the optimal value for the parameter \#samples.

\section{Functionality provided by FCD systems}

In this subsection it is investigated which functionality can be expected under optimal conditions $(100 \%$ penetration rate, $1 \mathrm{~s}$ sample interval). A major function is the estimation of current travel speed for a road segment. In scenario 1, simulations of the optimal conditions were performed for all 5 traffic densities (a,b,c,d,e), and the estimations were compared with the actual situation in the SUMO traffic simulation. Based on a visual inspection of all corresponding figures, it could be concluded that in all cases the estimation was very accurate.

The same experiments were performed for scenario 2, and some interesting effects were observed. First of all, the actual speed can fluctuate significantly in the proximity of traffic lights (Figure 3, segment 27 is immediately congested; segment 17 becomes congested after 4600 seconds). This means that in the context of accurate travel time estimation, the absolute value of the estimated speed close to traffic lights has limited value at one specific moment in time. However, when averaging out these values over a longer period of time (e.g. 10 times the lights' cycle length), it can be seen on Figure 3 that the average for a congested crossing (approx. 10 
$\mathrm{km} / \mathrm{h}$ ) is lower than in the non-congested case (approx. $40 \mathrm{~km} / \mathrm{h}$ ). This way it can be derived if a crossing is congested or not.

Two other important functionalities are the location determination of an incident and of the tail end of a traffic jam. To examine if these can be performed relying on FCD, the scenarios with heavy congestion (d) and with an incident (e) were analyzed both for the highway and the arterial setting. It was noticed that the tail end of a traffic jam and the location of an incident can be clearly identified in all scenarios. The results of the incident scenarios (e) are given in Figure 4 and Figure 5. In both cases, the estimated speeds for all segments are shown, for 4 different moments in simulation time: 1800, 2900, 4000 and 5100 seconds. The evolution of the traffic jam can clearly be distinguished. The location of the incident remains on exactly the same location, while the traffic jam itself becomes longer and longer. In both scenarios and for the different moments in time, the location of the incident and tail of the traffic jam as calculated by the FCD system was compared with the actual situation. In all cases there was an identical match. Similar results were obtained when the scenario without an incident but with heavy congestion was analyzed both for the highway as the arterial environment.

\section{Minimum requirements of a FCD dataset}

In this section the minimal requirements are determined for which a FCD system can provide the same performance as under optimal conditions. Three parameters are considered: penetration rate, sample interval and \#samples. These parameters cannot be optimized separately since they directly influence each other. An optimization strategy was defined in which penetration rate is considered to be the most important parameter. Hence it is minimized first. This is because it determines the number of vehicles that have to be equipped with GPS probes, significantly influencing the required initial investment and also the practical feasibility of the FCD deployment. The sample interval is maximized next since it determines the amount of data to be transmitted, one of the key elements of the operational cost. The parameter \#samples does not influence costs, but it can be used to increase the accuracy for a given combination of penetration rate and sample interval. It is optimized last in a fine-tuning phase. In every step of the optimization, the results under optimal FCD conditions (100\% penetration rate, 1 second sample interval, \#samples value of 5) are considered as the ground truth. 
A similar optimization could not be found in literature. Penetration rate and sample interval where researched before, but they are typically optimized independently as described in section 2. Only Nanthawichit et al. [10] took the interaction between these parameters into account. However, this study was limited to the highway environment, concluding with an optimal penetration rate of $4-5 \%$ in combination with a sample interval of 10 30 seconds. Because this penetration rate is significantly higher than the $1 \%$ found for highways by Chen \& Chien [9] and because of the lack a similar optimization study for the urban environment, it was chosen to investigate both environments in this paper. For each environment the five different types of traffic intensity are examined. The results are given in Table 1. Two conclusions of the table (scenario 1-b and 2-c) are illustrated in Figure 6 and Figure 7. All conclusions summarized in the table were derived from similar figures.

Table 1. Minimum requirements FCD

\begin{tabular}{llllll}
\hline Environment & Traffic density & Scenario nr & Penetration rate & Sample interval (s) & \#samples \\
\hline Highway & Night time & $1-\mathrm{a}$ & $1 \%$ & 20 & 5 \\
Highway & No congestion & $1-\mathrm{b}$ & $0.5 \%$ & 20 & 5 \\
Highway & Medium congestion & $1-\mathrm{c}$ & $0.5 \%$ & 60 & 2 \\
Highway & Heavy congestion & $1-\mathrm{d}$ & $0.5 \%$ & 20 & 5 \\
Highway & Incident & $1-\mathrm{e}$ & $1 \%$ & 20 & 5 \\
Arterial road & Night time & $2-\mathrm{a}$ & $10 \%$ & 1 & 2 \\
Arterial road & No congestion & $2-\mathrm{b}$ & $10 \%$ & 1 & 2 \\
Arterial road & Medium congestion & $2-\mathrm{c}$ & $5 \%$ & 10 & 2 \\
Arterial road & Heavy congestion & $2-\mathrm{d}$ & $1 \%$ & 30 & 2 \\
Arterial road & Incident & $2-\mathrm{e}$ & $5 \%$ & 120 & 2 \\
\hline
\end{tabular}

It is observed that on highways, the minimum requirement to maintain accuracy is a penetration rate of $1 \%$, with a sample interval of 20 seconds. This is feasible. On arterial roads this becomes a penetration rate of $10 \%$ with a sample interval of 1 second. This is unfeasible. However, in this environment not all absolute values are meaningful, as mentioned in section 4. Therefore a discrepancy between ground truth and estimated segment speeds is acceptable, as long as there is no loss of accuracy in the determination of heavily congested segments and the location of incidents and the end of traffic jams. This is supported with a penetration rate of $1 \%$ and a 
sample interval of 30 seconds. Based on the above, we define a penetration rate of $1 \%$ and a (conservative) sample interval of 10 seconds as the minimum FCD requirements.

\section{Reliability analysis}

Reliability is an important aspect of FCD systems. On the client and server side this can be controlled by ensuring the quality of the applied hardware and software, as already being done in many other information systems. However, there are some uncertainties regarding the reliability of the mobile data network. It is unknown if the proposed FCD system would lead to an overload of the network. In urban environments data networks are typically designed for higher data loads, applying smaller network cells to support the larger amount of users. On highways however, cells in general have a diameter of a few kilometres. In combination with high traffic intensities it could be possible that a large number of FCD vehicles will be connected to a single cell, possibly overloading that cell.

To investigate this uncertainty, a model is applied that was developed in previous work. It was originally designed to assess the impact of GPS based road charging systems on Belgian mobile data networks. It is clear that both scenarios have a strong resemblance. The model contains traffic measurement data for the entire Belgian highway network (spread over 150 locations). Based on a number of parameters, it calculates the load on the network cell for every location, for every hour of the day. Further details can be found in Vandenberghe et al. [19]. Here only the parameters are introduced which are of importance for the FCD scenario. The sample interval is defined by the parameter $c$. The parameter $d$ determines the distance between two transmissions of aggregated samples. The penetration rate is determined by the variable $p$. The length of a cell is given by $l$, the appropriate value for Belgium is $2.4 \mathrm{~km}$. The data overhead for the setup of a secure connection to the server is given by the parameter $O$. The byte size of a single sample is determined by $b$, for which 50 is an appropriate value in the FCD scenario.

Two techniques to minimize the load on the network are considered. The first one is to reduce the overhead for setting up a secure connection. For a normal SSL connection this is about 5000 bytes. Using a so called SSL restart handshake this could be reduced to 350 bytes. This type of handshake does not exchange security 
certificates, but relies on certificates which were stored after a normal handshake. These stored certificates can remain valid for a few days. The other technique is to increase the amount of useful data per transmission. In the FCD system that was suggested so far, a sample will be taken every 10 seconds and will be immediately transmitted to the FCD server. Storing a few samples before sending them in an aggregated manner will optimize communication efficiency. Inspired by [16], we propose to store 3 samples before transmitting them all at once, leading to a maximum delay of 30 seconds. To support this proposition, it was verified in different simulations that this delay is acceptable under all circumstances. Based on those techniques, 4 different scenarios are investigated, which are summarized in Table 2.

Table 2. Optimization scenarios in terms of data load

\begin{tabular}{lllllll}
\hline Scenario & $\boldsymbol{l}(\mathbf{k m})$ & $\boldsymbol{d}(\mathbf{s e c})$. & $\boldsymbol{c}(\mathbf{s e c})$. & $\boldsymbol{b}$ (bytes) & $\boldsymbol{O}$ (bytes) & $\boldsymbol{p}(\%)$ \\
\hline No aggregation - Normal SSL handshake & 2.4 & 10 & 10 & 50 & 5000 & 1 \\
No aggregation - Restart SSL handshake & 2.4 & 10 & 10 & 50 & 350 & 1 \\
Aggregation - Normal SSL handshake & 2.4 & 30 & 10 & 50 & 5000 & 1 \\
Aggregation - Restart SSL handshake & 2.4 & 30 & 10 & 50 & 350 & 1 \\
\hline
\end{tabular}

The results indicate that the load on the network cell can be neglected in all scenarios. Even without any optimization and on the busiest time of the day, a maximum of only 0.35 connections per second per cell is reached across the 150 different locations. This corresponds with a data load on the cell of just 14 kilobit per second (Figure 8). Although there is no need to implement the proposed data load optimizations from a reliability point of view, it can be derived from Figure 8 that they can have a significant influence on the total amount of data that needs to be communicated. Therefore it is advised to apply aggregation and the restart SSL handshake because of the positive influence on communication cost. In this case a single FCD vehicle transmits 500 bytes every 30 seconds. 


\section{Cost analysis}

In this section, four FCD scenarios are compared in terms of costs for the Belgian use case. The first scenario uses a dedicated hardware unit to collect and transmit FCD samples. The second option proposes a smartphone application to do the same. The third scenario is a reference to previous work which analyzed the rollout of a complete Intelligent Transportation System as a generic platform. In that case FCD collection could be part of the application set on the platform. This scenario is not elaborated upon in this paper, further details can be found in [20], but it is included in the scenario comparison in section 7.2. In the last scenario, data is purchased from companies which already acquire FCD through their own products (Personal Navigation Devices, probes for fleet management or personalized insurances, speed camera information systems, etc). As these prices are negotiated bilaterally, we can't calculate them. Instead, we will use the other scenarios to determine a reasonable cost.

\subsection{Cost elements}

The first cost element is the communication costs induced by the hardware unit. It can be derived from the results obtained in section 6 . There it was shown that 500 bytes are sent every 30 seconds. Based on Belgian traffic statistics, this corresponds with roughly $1.200 \mathrm{kB}$ of data each month. In the smartphone application scenario, this limited amount of data traffic is assumed to fall well within the limits of the users' mobile subscription and no cost is considered. For the dedicated unit, we foresee a monthly machine to machine subscription of 1-2 MB at a cost of 2.5 EUR (for a large quantity of SIM cards, based on previous experience of the authors).

A second cost element is the hardware component. For the dedicated hardware scenario, this unit is a relatively simple device that incorporates a GPS receiver, a UMTS interface, a controller and a plastic shell. Based on component prices derived from hardware sale sites $^{3}$, we estimate the direct cost at about 100-150 EUR. We expect a relatively high quantity of units produced, and therefore estimate the final cost at about 75 EUR due to economies of scale. In case of the smartphone scenario, we assume the GPS-coordinates and mobile connection to be already available. Still, a dedicated application will need to transmit coordinates when the user is driving the car, and not send anything when the user is not. It is unrealistic to expect the user to turn the application on 
and off at the right times, therefore we foresee a simple hardware unit that is installed inside the vehicle and connects to the smartphone through Bluetooth. When the smartphone application senses the device, it sends the corresponding ID along with the rest of the FCD sample to the central server. This Bluetooth device only consists of a Bluetooth module and a controller inside a plastic shell. We estimate the final cost at about 5 EUR (taking a large quantity into account). There is no hardware component for the third scenario.

Hardware installation is a third cost element. It consists of attaching the device to the vehicle (most likely underneath the vehicle for esthetical reasons) and connecting it to the power supply of the vehicle. We assume this will take about one hour of work, which can be performed when the car is taken to a garage for maintenance. Expected cost is then 45 EUR.

The following cost element is the design of the hardware unit. For the dedicated unit, we assume this will take 6 months of work for an engineer (costing $5.000 \mathrm{EUR} /$ month). For the Bluetooth device, we assume it will only be 3 months of work. To start mass production, we assume a commercial and technical person will need to establish contacts with Original Equipment Manufacturers. For the dedicated unit, we assume 6 months of work for both people (at $4.000 \mathrm{EUR} / \mathrm{month}$ each); while we use only 3 months of work for the Bluetooth device. Finally, we assume 20.000 EUR of expenses in both cases. We also foresee three months of work for a software programmer to create the smartphone application for Android and iPhone (at 4.000 EUR/month).

A fifth cost element is a financial incentive to stimulate drivers to install the system in their vehicle. The exact nature of this incentive (tax reduction, product, financial gift, etc) is irrelevant; we simply assume a one-time cost of 25 EUR per installed device.

The last element is the cost of the central FCD infrastructure. The incoming data stream will be rather limited in all scenarios, and it is assumed that two servers will suffice, with a cost of 1.500 EUR per year per server. Apart from the hardware, a team must also be ready to handle questions and complaints by users. In the dedicated hardware unit scenario, it is assumed that these will be very limited at one call per four years per user. For the smartphone application we assume twice that amount. An average call duration of 15 minutes, an average

\footnotetext{
${ }^{3}$ E.g. Farnell, www.farrnell.com
} 
occupancy rate of help desk employees of $66 \%$ and a wage of 35.000 EUR for a helpdesk employee lead to a cost of about 7.5 EUR per phone call.

In order to assess a reasonable data purchase cost, it is assumed that the total cost will be about $10 \%$ of the best alternative. This is based on the fact that prices cannot become much higher since a higher price would make the other solution more viable (more expensive but also more flexible). Competition between data providers will also push prices down. On the other hand, when prices would become any lower, the financial profit for the data owners would become negligible.

\subsection{Results}

The cost results are summarized in Table 3. These costs are the Net Present Value of 10 years of providing FCD, with a discount rate of $5 \%$. The generic ITS scenario is obviously more complex and expensive, but also more flexible and of more value to the users (such that they might pay for it, partially or wholly). The installed vehicle base evolves from $4 \%$ to $20 \%$ of the vehicles in Belgium, more than sufficient for the collection of FCD. The cost breakdown of this scenario is quite different from the cost breakdown of the other cases scenario (Vanhauwaert et al. [20]). Therefore only the total cost is included in Table 3. For the scenario relying on dedicated FCD hardware, we use the minimum of $1 \%$ installed vehicle base, while for the smartphone application and data purchase scenarios, it is assumed that the penetration will be higher than the minimum determined in section 5 . This is because it is expected that there will be a higher chance that data cannot be collected (e.g. smartphone or PND not turned on), or that the behaviour of a vehicle is biased (e.g. taxi's or trucks equipped with fleet management probes). As mentioned in section 7.1, cost of data purchase is derived from the cost of the best alternative (in this case the smartphone approach).

Table 3. Cost overview for the Belgian use case, including deduced data purchase cost (Net Present Value over 10 years, $5 \%$ discount rate)

\begin{tabular}{lrrr|r}
\hline Scenario & Generic ITS & Dedicated HW unit & Smartphone & Data purchase \\
\hline Penetration rate & $4 \%$ to 20\% & $1 \%$ & $1.50 \%$ & $2 \%$ \\
Cost (million EUR, 10 years) & 850 & 27.2 & 13.0 & 1.3 \\
Hardware & & 8 & 0.8 & \\
Installation & & 4.8 & 7.2 & \\
Incentive cost & 1.5 & 2.3 & 0 \\
Communication & & 12 & 0 & \\
Central servers & & 0 & 2.7 & \\
Helpdesk & & 0.9 & & \\
\hline
\end{tabular}


The results indicate that the options to acquire FCD vary strongly in terms of costs. We expect that acquiring the data with an own system will be significantly more costly than data purchase, which costs about 130.000 EUR per year in the Belgian use case. This requires that data providers are willing to sell the data. Moreover, the vehicle set of the data provider should be a good statistical representation of the entire traffic composition (e.g. not limited to only taxis or trucks). On the other hand, if there is a need for more than just FCD, the other scenarios offer more expansion possibilities.

\section{Conclusions and future work}

The feasibility of applying FCD to support dynamic traffic management was researched in this paper. Based on the obtained results, it is advised to target a FCD deployment with a penetration rate of $1 \%$ and a sample interval of 10 seconds. Such a system will be able to accurately determine the locations of incidents and the tail end of traffic jams on all road types. It will also be able to estimate travel times per road segment. For highways this estimation will be very accurate, for arterial and urban roads this is limited to the binary categorization into normal or congested traffic. From a reliability point of view, the influence of the FCD system on the mobile data network was the major point of concern. However, even without any optimizations the proposed FCD system causes a negligible load on the network. Hence no reliability issues are expected. To minimize communication costs it is recommended to aggregate 3 samples before transmission to the FCD server, and to apply the SSL restart handshake to setup the connection to the server. Taking the cost of the system further into account, the most efficient solution is to purchase FCD data from companies which already acquire such data through their own products (connected Personal Navigation Devices, probes for fleet management or personalized insurances, speed camera information systems, etc). In this case the estimated cost for the Belgian use case is 130.000 EUR per year.

Interesting future would be to refine the requirements for FCD data providers by replacing the deliberately simple traffic estimation algorithm with more sophisticated varieties based on their actual implementations. This would require them to share sensitive information regarding their operational deployments, but it is expected that the estimated costs could be lowered, making this solution even more attractive to policy makers. This is because these advanced algorithms will most likely be able to achieve equal accuracy with less data. Since the 
amount of monthly communicated data is quite limited (1.2 MB), we would propose to lower the required penetration rate. This will decrease the total cost of the system.

\section{Acknowledgements}

The authors would like to acknowledge the Flemish Policy Research Centre Mobility \& Public Works, track Traffic Safety for funding this work.

\section{References}

[1] Quiroga, C.A. and Bullock, D.: 'Travel time studies with global positioning and geographic information systems: an integrated methodology', Transportation research part c - emerging technologies, 1998, 6, (12), pp. 101-127

[2] De Fabritiis, C., Ragona, R. and Valenti, G.: 'Traffic estimation and prediction based on real time floating car data', Proc. 11th International IEEE Conference on Intelligent Transportation Systems, Beijing, China, October 2008, pp. 197-203

[3] Li, X., Shu, W., Li, M., Huang, H.-Y., Luo, P.-E. and Wu, M.Y.: 'Performance evaluation of vehicle-based mobile sensor networks for traffic monitoring', IEEE Transactions on Vehicular Technology, 2009, 58, (4), pp. $1647-1653$

[4] Shi, W. and Liu, Y.: 'Real-time urban traffic monitoring with global positioning system-equipped vehicles', IET Intelligent Transport Systems, 2010, 4, (2), pp. 113-120

[5] Dion, F. and Rakha, H.: 'Estimating dynamic roadway travel times using automatic vehicle identification data for low sampling rates', Transportation research part B - Methodological, 2006, 40, (9), pp. 745-766

[6] Bar-Gera, H.: 'Evaluation of a cellular Phone-based system for measurements of traffic speeds and travel times: a case study from Israel', Transportation Research part C: Emerging Technologies, 2007, 15, (6), pp. $380-391$ 
[7] Herrera, J. C., Work, D.B., Herring, R., Ban, X.G., Jacobson, Q. and Bayen, A. M.: 'Evaluation of traffic data obtained via GPS-enabled mobile phones: the Mobile Century field experiment', Transportation research part C - Emerging Technologies, 2010, 18, (4), pp. 568-583

[8] Breitenberger, S., Grüber, B., Neuherz, M. and Kates, R.: 'Traffic information potential and necessary penetration rates', Traffic Engineering and Control, 2004, 45, (11), pp. 396-401

[9] Chen, M. and Chien, S.I.J.: 'Dynamic freeway travel time prediction using probe vehicle data: link-based vs. path-based', Proc. 80th Annual Meeting of the Transportation Research Board, Washington DC, USA, Jan. 2001, pp. 1-14

[10] Nanthawichit, C., Nakatsuji, T. and Suzuki, H.: 'Dynamic estimation of traffic states on a freeway using probe vehicle data', Journal of Infrastructure Planning and Management, 2003, 730, pp. 43-54

[11] van Lint, J.W.C. and Hoogendoorn, S.P.: 'The technical and economic benefits of data fusion for real-time monitoring of freeway traffic: preliminary results and implications of a study with simulated data', Proc. $11^{\text {th }}$ World Conference on Transport Research, Berkeley, USA, June 2007, pp. 1-15

[12]Hong, J., Zhang, X., Wei, Z., Li, L. and Ren, Y.: 'Spatial and temporal analysis of probe vehicle-based sampling for real-time traffic information system', Proc. 2007 IEEE Intelligent Vehicles Symposium, Istanbul, Turkey, June 2007, pp. 1234-1239

[13] Mu, B., Hu, J. Zhao, T. and Zhang, Y.: 'Evaluating the performance of link travel time estimation based on floating car data', Proc. 2010 International Conference on Optoelectronics and Image Processing, Haikou, China, November 2010, pp. $504-508$

[14]Cheu, R.L., Xie, C. and Lee, D.-H.: 'Probe vehicle population and sample size for arterial speed estimation', Computer-Aided Civil and Infrastructure Engineering, 2002, 17, (1), pp. 53-60

[15]Fontaine, M.D. and Smith, B.L.: 'Investigation of the performance of wireless location technology-based traffic monitoring systems', Journal of transportation engineering, 2007, 133, (3), pp. 157-165

[16] Jiang, G., Gang, L. and Cai, Z.: 'Impact of probe vehicles sample size on link travel time estimation', Proc. 2006 IEEE Intelligent Transportation Systems Conference, Toronto, Canada, September 2006, pp. 505-509 
[17]Dion, F., Robinson, R. And Oh, J.-S.: 'Evaluation of usability of IntelliDrive probe vehicle data for transportation systems performance analysis', Journal of Transportation Engineering, 2011, 137, (3), pp. 174-183

[18] Cohn, N.: 'Real-time traffic information and navigation', Transportation Research Record: Journal of the Transportation Research Board, 2009, 2129, pp. 129-135

[19] Vandenberghe, W., Carels, D., Moerman, I., Demeester, P., Bergs, J., Van de Velde, E., Van den Wijngaert, N., Blondia, C. and Dedene, N.: 'Impact of introducing road charging on supporting mobile data networks', Proc. 9th International Conference on Intelligent Transport Systems Telecommunications, Lille, France, October 2009, pp. 19-24

[20] Vanhauwaert, E., Verbrugge, S., Vandenberghe, W., Pickavet, M. and Demeester, P.: 'Realistic cost estimation of an intelligent transportation system roll-out', Journal of the Institute of Telecommunications Professionals, 2011, 5, (1), pp. 39-45 


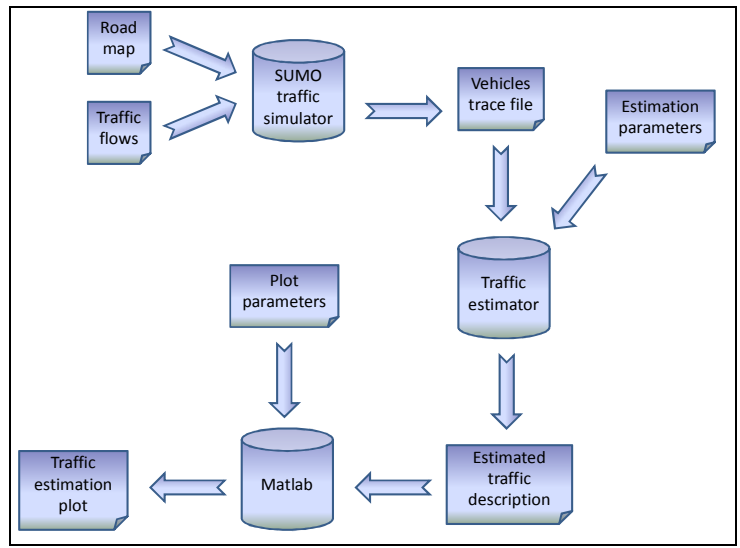

Figure 1. FCD simulation platform 


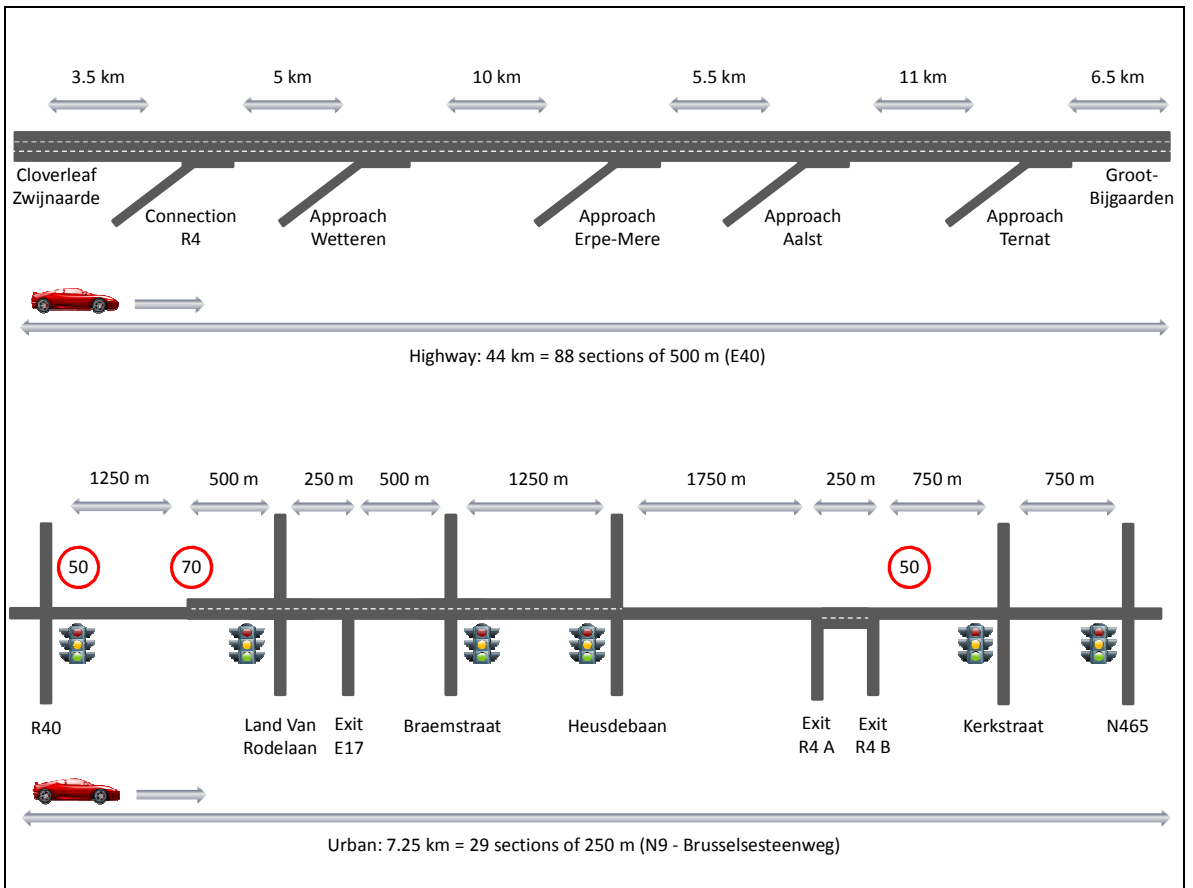

Figure 2: Simulated scenarios: scenario 1 (top) represents a highway environment, scenario 2 (bottom) represents an urban setting 


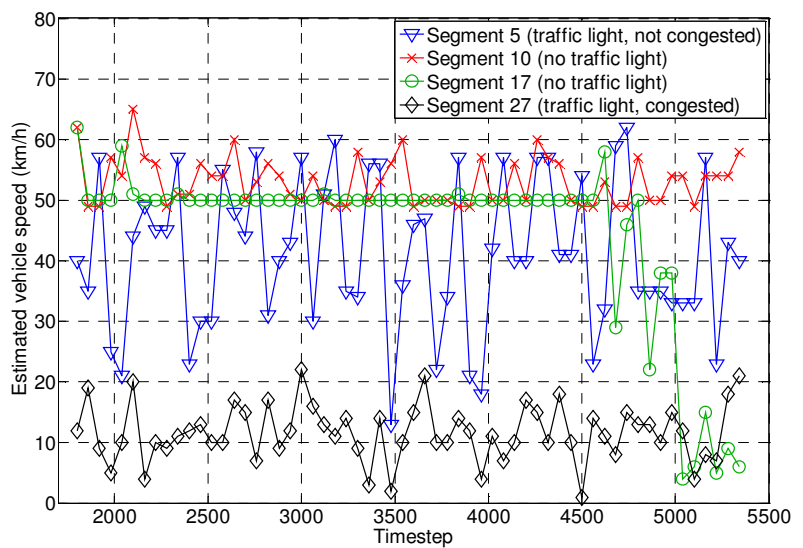

Figure 3. Fluctuations of speed estimations close to traffic lights (Scenario 2-d, optimal FCD conditions) 


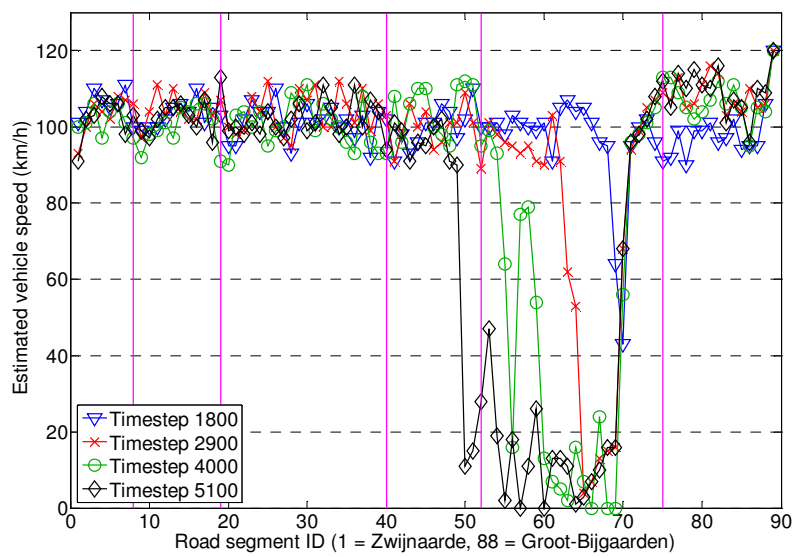

Figure 4. Incident detection on highway (Scenario 1-e, optimal FCD conditions) 


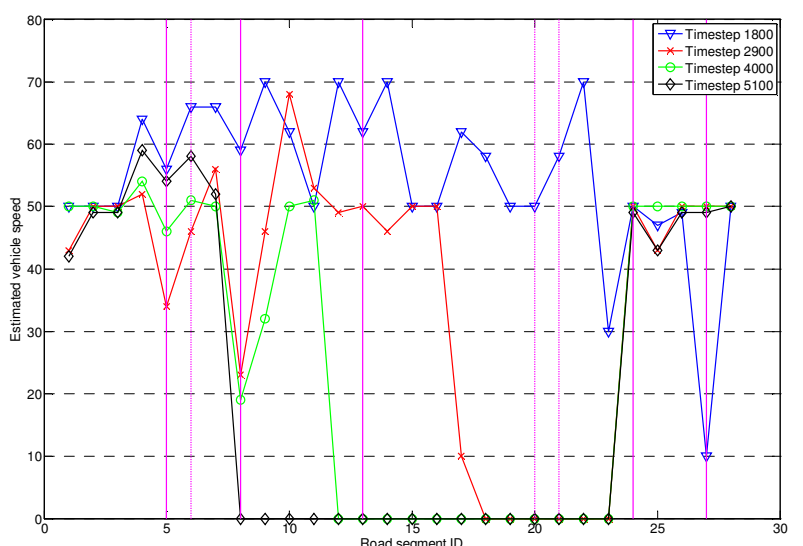

Figure 5. Incident detection on arterial road (Scenario 2-e, optimal FCD conditions) 


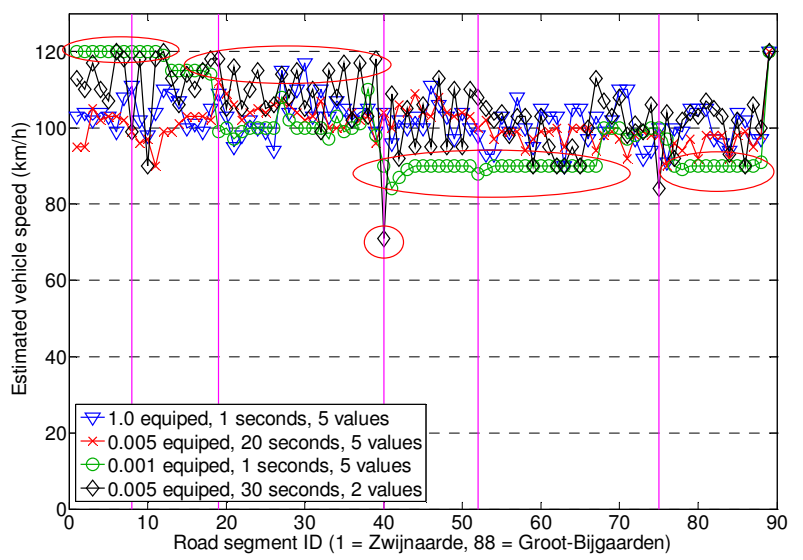

Figure 6. Minimum FCD requirement for scenario $1-b$ is $0.5 \%$ penetration and 20 s sample interval (smaller penetration or larger interval loses accuracy) 


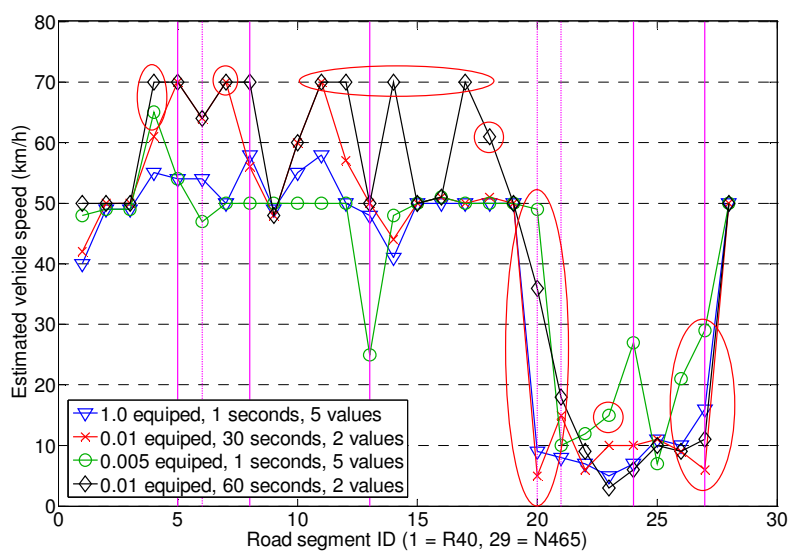

Figure 7. Minimum FCD requirement for scenario $2-d$ is $1 \%$ penetration and $30 \mathrm{~s}$ sample interval (smaller penetration or larger interval loses accuracy) 


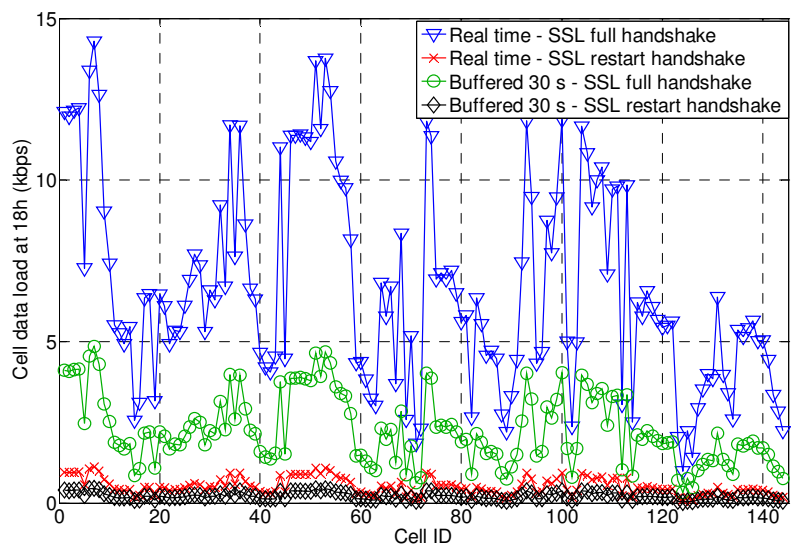

Figure 8. Transferred FCD data (kilobit) per network cell per second at $18 \mathrm{~h}$ (for 150 different locations on Belgian highways) 